\title{
Sampling with Finite Rate of Innovation: Channel and Timing Estimation for UWB and GPS
}

\author{
Julius Kusuma $^{1}$, Irena Maravić ${ }^{2}$ and Martin Vetterli ${ }^{2,3}$ \\ kusuma@mit.edu, \{irena.maravic, martin.vetterli\}@epfl.ch
}

\begin{abstract}
In this work, we consider the problem of channel estimation by using the recently developed theory for sampling of signals with a finite rate of innovation [1]. We show a framework which allows for lower than Nyquist rate sampling applicable for timing and channel estimation of both narrowband and wideband channels. In certain cases we demonstrate performance exceeding that of algorithms using Nyquist rate sampling while working at lower sampling rates, thus saving power and computational complexity.
\end{abstract}

\section{INTRODUCTION}

$\mathbf{C}$ HANNEL estimation is a rich area for research and development in wireless communication, ranging and timing, etc. Most current methods perform the estimation by using a matched filter when the pulse shape is known but the timing is unknown, or a training sequence when the pulse shape is unknown.

We have recently developed a framework which takes advantage of transform techniques to be able to perform perfect reconstruction of signals by sampling below the traditional Nyquist rate [1]. The critical sampling rate required for perfect reconstruction in our framework is the signal innovation rate. In subsequent work this same concept has been extended to lowering the required sampling rate of UWB (Ultra WideBand) receivers [2], [3], [4], and CDMA receivers [5].

By lowering the sampling rate below that traditionally believed to be the critical rate, we can significantly lower the power consumption of the A/D receivers, and allow for highresolution estimation of parameters with very simple hardware. In effect, we use an algorithmic solution to improve the hardware specifications in terms of complexity and power.

\section{Problem Statement}

Many communication systems require the receiver and/or the transmitter to know the channel impulse response. Other applications, most notably GPS and UWB-based ranging systems, require very accurate timing estimation [6]. All of these systems typically either have very high sampling frequencies, or use very complex hardware systems to enable highly-accurate timing estimation.

Consider the following simple example. Let the signal $x(t)$ be a (noisy) sum of delta functions with time delays $\left\{t_{k}\right\}_{k=0}^{K-1}$ :

$$
x(t)=\sum_{k=0}^{K-1} a_{k} \delta\left(t-t_{k}\right)+n(t) .
$$

1) Lab. for Information and Decision Systems, MIT; 2) LCAV, École Polytechnique Fédérale de Lausanne, Switzerland; 3) Dept. of EECS, U.C. Berkeley
The Fourier transform of this signal is given by:

$$
X(\omega)=\sum_{k=0}^{K-1} a_{k} \exp \left(j \omega t_{k}\right)+N(\omega) .
$$

In this case, we have converted the problem of estimating the time delays into the problem of estimating the frequency components of superimposed complex exponentials.

The problem of frequency estimation is well-studied: there exists a rich body of literature on both the theoretical limits and efficient algorithms for reliable estimation. See Stoica and Moses [7] for a review of the different algorithms. There is an especially attractive class of algorithms called the superresolution estimators, which has been extensively studied and applied to many problems from classical frequency estimation to angle-of-arrival estimation in antenna arrays. The key feature of this class of algorithms is that they perform very well even with modest numbers of samples. There are also statespace methods which perform well with very few numbers of samples [8]. In our problem formulation, taking fewer samples in the frequency domain is equivalent to taking fewer samples in the time domain.

\section{A. Delay Estimation}

First we consider the problem of delay estimation of UWB and CDMA-type signals, such as those found in GPS systems. UWB for indoor positioning has been proposed by various parties, its attractive feature being the potential to obtain very highresolution timing estimates, which in turn gives very accurate positioning performance. Various methods have been proposed and they can be broadly classified into digital- and analogoriented systems.

In a digital-oriented system, the receiver uses a very highspeed $\mathrm{A} / \mathrm{D}$ converter operating in the gigahertz range. In an analog-oriented system, the receiver uses a correlator-type of detector implemented in the analog domain. To allow for accurate timing estimation for example, a solution proposed by Aetherwire implements a cascade of correlators, called the Time-Integrating Correlator (TIC) (for references, see for example http://www.aetherwire.com). Both of these solutions consume a lot of power, and for the latter case, a significant fraction of resources in the circuit implementation. In fact, in many implementations the TIC alone can take up approximately $30 \%$ of the total circuit area!

The deployment of such schemes also present some additional issues. Many parties have proposed a CDMA-modulated 
UWB system for localization systems, where several transmitters are placed at known locations to allow a mobile receiver to estimate its location similar to the operation of GPS receivers. It is well-known that the multipath environment will induce multiaccess interference at the receiver, and that power control is needed for improved receiver performance due to what is called the near-far problem. This introduces additional complications when there are many mobile users in the system, plus it requires the availability of an uplink channel for the control loop. We will demonstrate by simulation that our proposed scheme is near-far resistant.

\section{B. Channel Estimation}

We are now ready to graduate to the channel estimation problem. We consider three channel models shown in Fig. 1:

1) Stream of impulses given by:

$$
h(t)=\sum_{l=0}^{L-1} a_{l} \delta\left(t-t_{l}\right) .
$$

2) Stream of pulses given by:

$$
h(t)=\sum_{l=0}^{L-1} a_{l} p\left(t-t_{l}\right) .
$$

3) Stream of different pulses given by

$$
h(t)=\sum_{l=0}^{L-1} a_{l} p_{l}\left(t-t_{l}\right)
$$

The first two models are popularly used to model outdoor fading channels and sparse scatterers, found for example in GPS applications and cellular radio. The last model is a more accurate model for UWB, where different incoming paths are subjected to different frequency-selective attenuations [9]. UWB signals occupy a very broad regime of spectra by definition [3], [4]. Due to this property, many environments provide a wealth of resolvable multipath [10].

The models of (3) and (4) are effective for modeling certain types of channels, where $p(t)$ is the antenna transmit pulse shape. These simple models are in reality less realistic than one may be led to believe, particularly for UWB signals. Since UWB signals are very broadband, the different propagation paths undergo different frequency-selective mitigation as they reflect off objects or penetrate through them. Therefore a more realistic model is given by (5) where now the received pulses $p_{k}\left(t-t_{k}\right)$ are different for different propagation paths.

Due to the apparent coupling of the angles of arrival with the pulse shapes, in a previous work [11] Cramer, Scholtz and Win used an antenna array to achieve spatio-temporal separation of the received signal components. The measurements are processed by the CLEAN algorithm, which was originally developed for ultra-wideband space telemetry applications.

We are interested in low-complexity, possibly even singleantenna applications where the receiver can separate the different incoming pulses in (3). In the next section we will show that we can achieve this using a single antenna. However, we should note that the framework presented here, following [5], is extensible to the case given by (5) using multiple receive antennas.
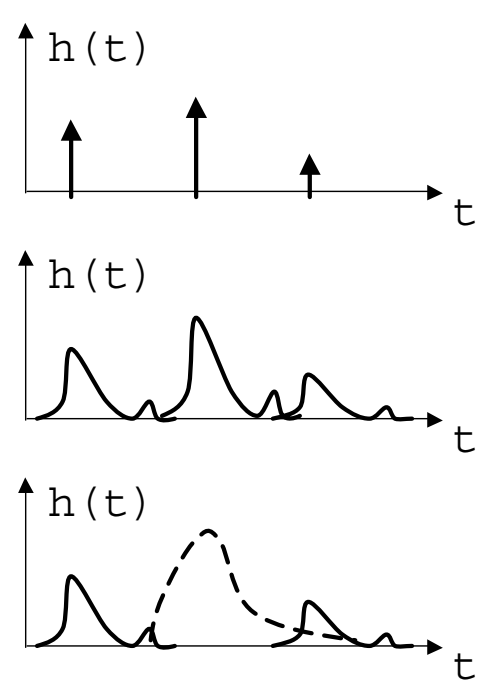

Fig. 1. Channel models for channel estimation: (a) Narrowband channel model, (b) Wideband channel model with few scatterers, (c) UWB channel model with many scatterers.

\section{Algorithm}

We propose a method for channel and timing estimation which uses very low-rate uniform sampling and well-developed estimation algorithms. The idea is based on [5].

First we derive the Fourier transform of a more general wideband channel model of (5):

$$
r(t)=\sum_{k=0}^{K-1} \sum_{l=0}^{L-1} g_{k}^{(l)}\left(t-t_{k}^{(l)}\right)+z(t),
$$

with Fourier series coefficients given by the following vector:

$$
R[m]=\sum_{k=0}^{K-1} \sum_{l=0}^{L-1} G_{k}^{(l)}[m] e^{j \omega t_{k}^{(l)} m}+Z[m],
$$

where $r(t)$ is the received signal, $k$ indexes the different pulses and $l$ indexes different time delays of the pulses, $g_{k}(t)$ denote the different pulses including the amplitude and phase terms, $t_{k}^{(l)}$ denotes the time delays, and $z(t)$ denotes receiver noise. The corresponding Fourier representations are in uppercase variable names. In this model we essentially capture different pulses and different paths taken by these different pulses. This is applicable to multiuser communication systems [5].

Suppose now that all the $G_{k}^{(l)}[m]$ terms have degree $\leq D-1$. Then we can write, for each $k$ and $l$, the following vector:

$G_{k}^{(l)}[m]=\sum_{r=0}^{D-1} g_{r, k}^{(l)} m^{r}=g_{0, k}^{(l)}+g_{1, k}^{(l)} m+\ldots+g_{D-1, k}^{(l)} m^{D-1}$

In general, $\left\{g_{r, k}^{(l)}\right\}$ are complex. Ignoring noise, using this representation in the Fourier representation of the received signal in (7), for fixed $k$ we obtain the following vector:

$$
R_{k}[m]=\sum_{l=0}^{L-1} \sum_{r=0}^{D-1} g_{r, k}^{(l)} m^{r} e^{j \omega t_{k}^{(l)} m}
$$


Now we can find an annihilating filter for the equation above, similar to the approach of [1]:

$$
H_{k}(z)=\left(1-e^{-j \omega_{0} t_{k}^{(l)}} z^{-l}\right)^{D}
$$

where only the time delays $\left\{t_{k}^{(l)}\right\}$ appear in the filter terms. Intuitively, $g_{d, k}^{(l)}$ appears as "modulated message" in the frequencydomain representation of the received signal, which carrier frequency is $t_{k}^{(l)}$. By suppressing the carrier frequencies we cancel out the signal, without requiring that we know what the message really is. Therefore we can apply standard algorithms for frequency estimation to find the values of $\left\{\hat{t}_{k}^{(l)}\right\}$.

Once we have computed the annihilating filter by computing the nullspace of $R[m]$, we can then solve the Vandermonde system given in (7) to find the pulse coefficients $\left\{\hat{g}_{d, k}^{(l)}\right\}$. Quite interestingly, there are current methods which also use polynomial aproximation in the frequency domain for equalization of OFDM systems [12]: the approach of Meyr et.al. is to place training symbols in the time-frequency tiles (for example, in a latin squares arrangement). The receiver then estimates the remainder of th channel coefficients by using interpolating filters. The algorithm is given as follows:

1) Calculate the Fourier tranform of the received signal $R[m]$.

2) Form the correlation matrix of $R[m]$.

3) Calculate the nullspace of the correlation matrix to get $\left\{\hat{t}_{k}\right\}$.

4) Solve for the coefficients of $\left\{\hat{g}_{r, k}^{(l)}\right\}$ in the frequency domain, given $\left\{\hat{t}_{k}\right\}$, by solving the Vandermonde system.

We show the system in Fig. 2. We note several appealing features of our framework:

1) Low sampling rate, which corresponds to significantly lower power consumption.

2) Robustness to fading environments and filter characteristics.

3) Use of well-studied estimation algorithms.

\section{Simulation Results}

We simulated our algorithm for several scenarios mentioned in the previous parts: UWB timing performance in an indoor environment and CDMA in an outdoor environment. The former models emerging home positioning applications for UWB and the latter models the classical GPS estimation problem for Line-of-Sight situations.

\section{A. UWB indoor timing performance}

We simulated the performance of the proposed system for UWB environments, using the recently developed channel model of [13], operating in the $4.375-5.625 \mathrm{GHz}$ frequency range. We assume a distance of 10 meters, and generated 10 random channel outcomes for simulation. In our system we model the transmitted signal as a delta function. A more realistic model would require knowledge of the transmit and receive filters, which in turn depends on the hardware design. This is left for further work.

We show the performance results in Fig. 3 in terms of MSE (Mean-Square Error) versus received SNR averaged for different uniformly distributed timing arrivals and channel outcomes. To compare with the method proposed by some companies (for example, Aetherwire), we assume that the pulses are modulated with a 1023-chip PN sequence. The error is normalized to the chip duration $T_{c}$, and plotted for different values of a sampling rate ( $R_{c}$ is the chip rate).

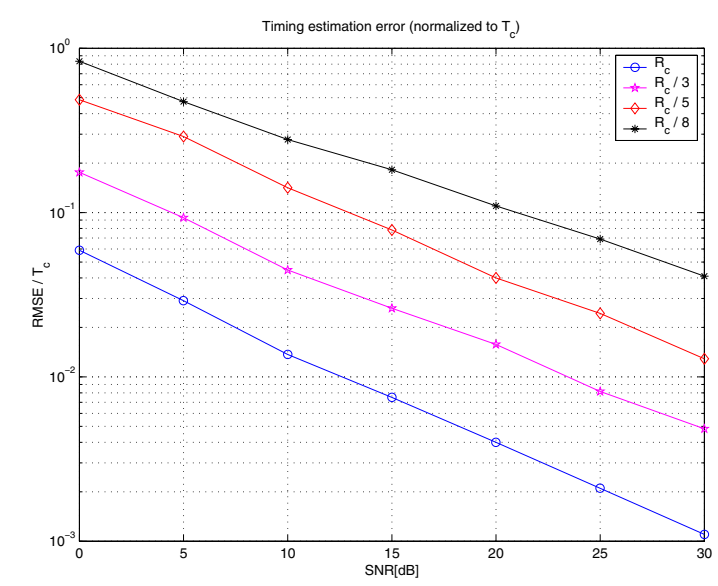

Fig. 3. Log MSE vs. SNR for same values of sampling rates.

As suggested by the Cramér-Rao bound for the estimation error of the frequency of complex sinusoids, the results vary as a function of the pulse location within the observation window: locations closer to the edges obtain better performance. This suggests an iterative strategy for accurate timing estimation, by which we move the observation window to place the expected pulse location closer to the edges of the window.

From our simulations we also note that the developed framework is robust against frequency-selective fading: it can be seen that the linear filter channel acts as a window on the frequencydomain representation of the received signal.

\section{B. CDMA outdoor timing performance}

In this set of simulations, we consider a CDMA signal modulated by a 511-long PN sequence ${ }^{1}$. This system gives superior performance compared to subchip-sampled systems due to the availability of super-resolution methods which solves the timing estimation problem in the frequency domain. The results are given in Fig. 4 for AWGN channels and Fig. 5 for fading channels.

We also demonstrate the effectiveness of the algorithm in a multi-user scenario for CDMA communication and localization applications in Fig. 4(b) for an AWGN channel. This scheme is also near-far resistant: we show this in Fig. 5(a). Finally, we simulate the performance of the scheme in a multipath fading channel and give the simulation results in Fig. 5(b).

\footnotetext{
${ }^{1}$ This result is derived in [5]
} 


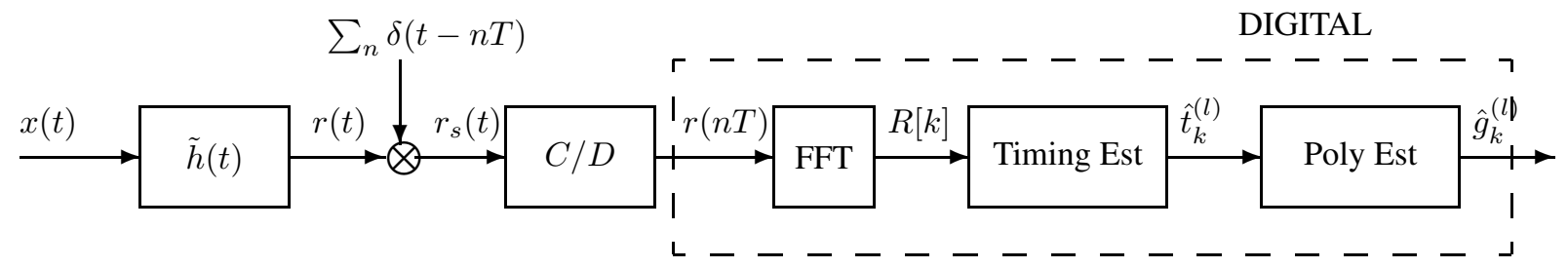

Fig. 2. System block diagram.

\section{Channel Estimation Performance}

Next we show the performance for the estimation of $\left\{g_{r, k}^{(l)}\right\}$ if we approximate smooth functions such as Gaussian, first derivative Gaussian, etc. using order-8 polynomials in our Vandermonde system in Fig. 6. The quality of the estimation can be improved by either increasing the sampling rate, or by allowing a larger-degree polynomial fit in the model.

\section{Computational Complexity}

We now discuss the computational complexity of low-rate channel/timing estimation framework. Let $M$ be the number of samples per symbol and $L$ be the number of users in the multi-user case. The most computationally intensive part of our system is the singular-value decomposition algorithm, which is part of the super-resolution method. This requires $\mathcal{O}\left(M^{3}\right)$ operations for each user in the system. If we use an annihilating filter method instead, we can reduce this to $\mathcal{O}\left(M^{2}\right)$ operations for each user in the system.

In contrast, matched-filter methods require $\mathcal{O}\left(F^{2} N^{2}\right)$ operations, where $F$ is the oversampling factor beyond the chip rate, and $N$ is the spreading factor. The resolution of the matched filter methods are usually limited by the chip duration. For example, consider the performance given in Fig. 4(a) where the spreading factor is 511 . At $5 \mathrm{~dB}$ SNR, by using only 1 sample for every 10 chip, which totals $\approx 50$ samples in a frame, we already achieve an error performance 0.7 times the chip duration. In comparison, the matched filter approach requires sampling at higher than the chip rate. We have thus demonstrated gains not only in performance, but also in the number of computations needed, in addition to using very low sampling rate.

\section{CONCLUSIONS AND DiscusSiOnS}

In this work we have shown that the new framework of sampling at the rate of innovation is an effective method for channel and timing estimation. In many cases, we note superior performance even to current methods which require sampling at or above the Nyquist rate, while we use a sub-Nyquist sampling. We demonstrated the effectiveness for wideband systems, and for localization applications such as GPS. For wideband channel estimation, we give a framework which allows for identification of more complex and realistic channel models without requiring the use of antenna arrays and their associated processing techniques. For timing applications, we give a framework which gives superior performance to that of conventional timing estimation methods.
Our framework takes advantage of well-developed theories and practice of frequency estimation, and uses efficient algorithms affording much lower required sampling rate, and in turn lower complexity and power consumption. Energy consumption scales quadratically with A/D Converter sampling rate and with processor speed. Therefore the methods proposed here promise significant reduction of power consumption. Moreover, this is consistent with current trends in moving more towards digital implementation in circuits and systems. The same framework can be extended to incorporate multiple antenna elements, as shown in [5].

\section{REFERENCES}

[1] M. Vetterli, P. Marziliano, and T. Blu, "Sampling signals with finite rate of innovation," IEEE Transactions on Signal Processing, vol. 50, pp. 1417 1428, June 2002.

[2] J. Kusuma, A. Ridolfi, and M. Vetterli, "Sampling of communication systems with bandwidth expansion," in Proc. of the IEEE Conference on Communication, pp. 1601 - 1605, April 2002.

[3] M. Z. Win and R. A. Scholtz, "Impulse radio: How it works," IEEE Communication Letters, vol. 2, pp. 36-38, February 1998.

[4] M. Z. Win and R. A. Scholtz, "Ultra-wide bandwidth time-hopping spread-spectrum impulse radio for wireless multiple-access communications," IEEE Transactions on Communications, vol. 48, pp. 679-691, April 2000.

[5] I. Maravić and M. Vetterli, "Digital DS-CDMA receivers working below the chip rate: Theory and design," IEEE Transactions on Communications, 2002. In review.

[6] G. Strang and K. Borre, Linear Algebra, Geodesy and GPS. Wellesley, MA: Wellesley - Cambridge, 1997.

[7] P. Stoica and R. Moses, Introduction to Spectral Analysis. Englewood Cliffs, NJ: Prentice-Hall, 1997.

[8] S. Y. Kung, K. S. Arun, and D. V. B. Rao, "State-space and singular-value decomposition-based approximation methods for the harmonic retrieval problem," Journal of the Optical Society of America, vol. 73, pp. 1799 1811, December 1983.

[9] M. Win, R. Scholtz, and M. Barnes, "Ultra-wide bandwidth signal propagation for indoor wireless communications," in Proc. IEEE ICC, pp. 5660, April 1997.

[10] M. Z. Win and R. A. Scholtz, "Characterization of ultra-wide bandwidth wireless indoor communications channel: A communication theoretic view," IEEE Journal on Selected Areas in Communications, vol. 20, pp. 1613-1627, December 2002. Invited Paper.

[11] R. J.-M. Cramer, R. A. Scholtz, and M. Z. Win, "Evaluation of an ultra wide-band propagation channel," IEEE Trans. on Antennas and Propagation, vol. 50, pp. 561 - 569, May 2002.

[12] H. Meyr, M. Moeneclaey, and S. A. Fechtel, Synchronization, Channel Estimation, and Signal Processing, Volume 2, Digital Communication Receivers. New York, NY: Wiley, 1997.

[13] W. Turin, R. Jana, V. Tarokh, S. Ghassemzadeh, and C. Rice, "Autoregressive modeling of an indoor UWB channel," in Proc. of the IEEE Conf. on UWB Technology, pp. 71 - 74, 2002. 


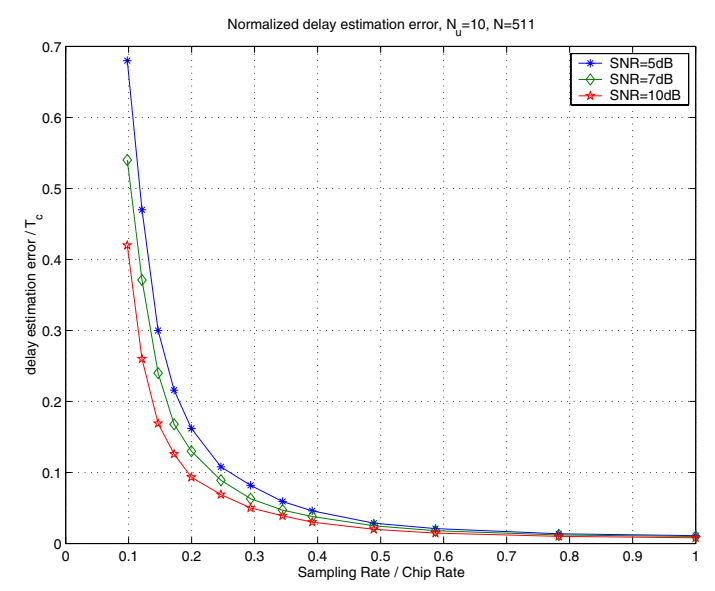

(a) Average timing synchronization error (normalized to $T_{c}$ ) in the multiuser case (10 users) vs. sampling rate in AWGN.

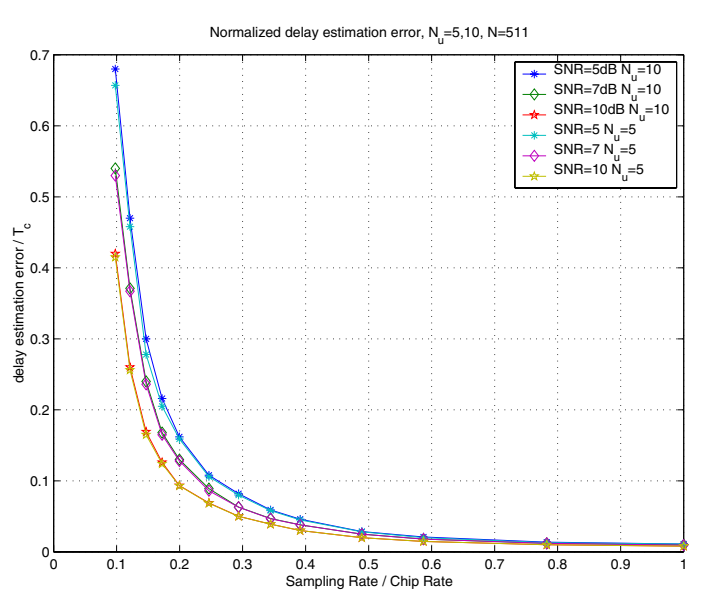

(b) Comparison of the timing synchronization errors in non-fading channels for systems with 5 and 10 users

Fig. 4. Timing performance for CDMA signals.

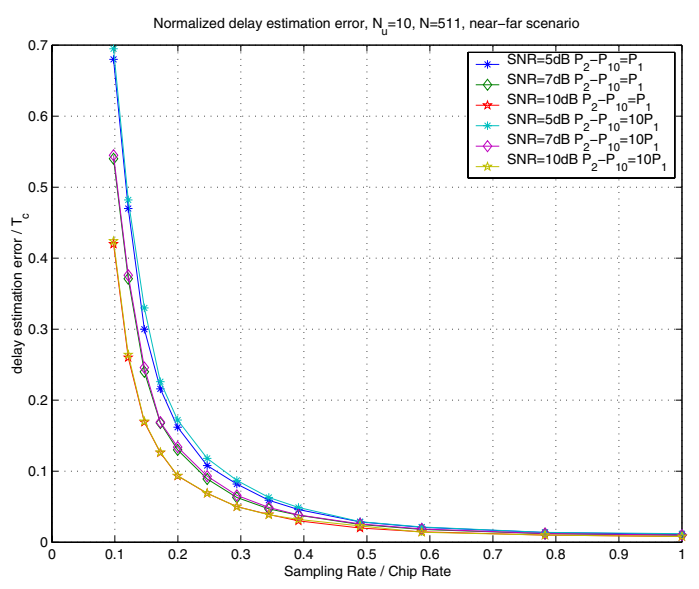

(a) Timing synchronization errors for a system with 10 users in the near-far case.

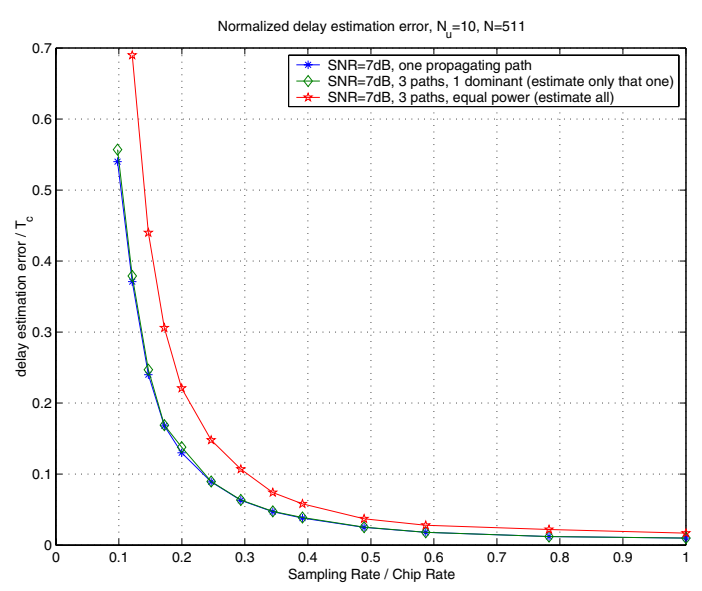

(b) Comparison of timing estimation errors for the system with 10 users is single-path and multipath channels (3 propagation paths for each user)

Fig. 5. Timing performance for CDMA signals.

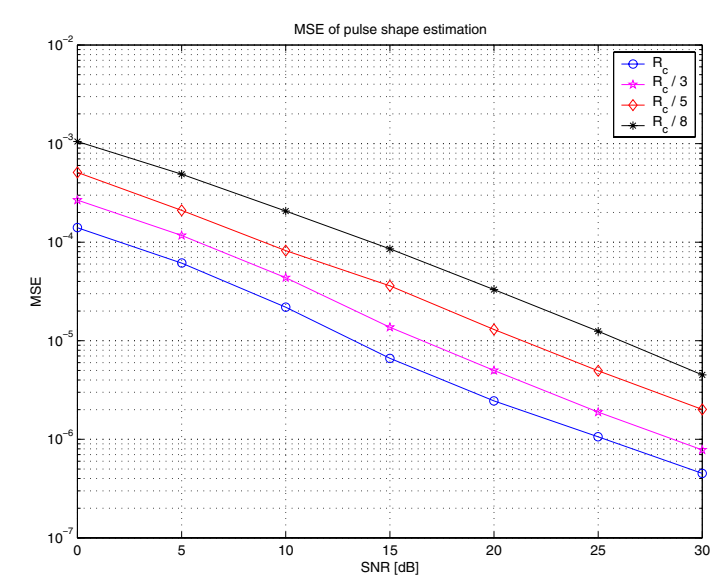

Fig. 6. Log MSE vs. SNR for approximation of smooth functions using order 8 polynomials in the Vandermonde system. 